\title{
A Molecular Dynamic Study on Nonlinear Vibration Behaviors of Fe
}

\section{Nanowires}

\author{
Zhuoqun Zheng ${ }^{1}$, Eric $\mathrm{Li}^{2}$, Nan Ding ${ }^{3}, \dagger \mathrm{Xu} \mathrm{Xu}^{1}$ \\ ${ }^{1}$ College of Mathematics, Jilin University, 2699 Qianjin Street, Changchun, 130012, China \\ ${ }^{2}$ Department of Mechanical and Automation Engineering, The Chinese University of Hong Kong, Hong Kong, \\ China \\ ${ }^{3}$ School of Management Science and Information Engineering, Jilin University of Finance and Economics, \\ Changchun 130117, China \\ †Corresponding author: xuxu@jlu.edu.cn
}

\begin{abstract}
In this paper, vibration behaviors of Fe nanowires are investigated by using the large scale Molecular Dynamics (MD) simulations. It is observed that the vibration frequency of nanowires rises slightly and nonlinearly with the increase of initial actuation amplitude. Based on the atomic arrangement, a discrete spring-mass model is developed. Its nonlinear elastic relation is used to explain this phenomenon. In addition, Fe nanowires with different lengths and heights show different vibration properties in this work. The ratio between the length $(L)$ and the height $(h)$ of nanowires has a significant influence on vibration behaviors. The vibration properties of nanowires can be explained by the Euler-Bernoulli model when the ratio is relatively large, while they can be illustrated by the Timoshenko model when the ratio is relatively small.
\end{abstract}

Keywords: nonlinear vibrations, Fe nanowires, molecular dynamics, initial actuation, length height ratio, spring-mass model.

\section{Introduction}

Nanowires have been widely investigated with the rapid development of nano science and technology. Due to their unique electronic, thermal, mechanical and optical properties, nanowires have been widely applied as active components of nanoelectromechanical systems (NEMS), such as force and pressure sensing [1, 2], nanowire-nanopore sensors [3], field effect transistor [4], lithium battery anodes [5] and other devices [6-8]. These NEMS utilize the nanowire as a resonating beam, in which the nanowire vibrates continuously at or near its 
resonant frequency. In addition, the changes in local environment including force, pressure or mass can be detected by the corresponding changes in the resonance frequency of the nanowire [9]. Therefore, it is of great significance to study the nanowire's mechanical properties under vibration.

Recently, several studies on the nanowire's vibrational behaviors and properties have been done, including the experiments [10-13], theoretical modeling [14-20] and computational simulations [21-29]. Some researchers focus on the influence of the nanowire's material. Copper, silver, gold and silicon nanowires have been investigated on their vibrational properties respectively [21, 24-28, 30]. Two-dimensional vibration of nanowire is an interesting and tough problem. Conley et al. have studied it theoretically [14] and Gil-Santos et al. have used it for mass sensing and stiffness spectroscopy [6]. In the nanowire's two-dimensional vibration, beat phenomenon is a special physical characteristic, which was reported and investigated recently [21, 22, 31, 32]. However, little attention has been paid to another tough and useful problem - the nonlinear vibration behaviors of nanowires, especially the Fe nanowires. Additionally, the initial actuation and nanowires' length height ratio are rarely considered in the study on nonlinear vibration behaviors of nanowires. Therefore, it is necessary to investigate the mechanism of the nonlinear vibration behaviors with consideration of the above factors.

Therefore, a fundamental numerical and theoretical study of nonlinear vibration behaviors of Fe nanowires is presented in this work. We investigate the nonlinear vibration behaviors of Fe nanowires in two aspects: initial actuation amplitude and nanowires' length height ratio. Based on the atomic structure, a valid discrete spring-mass model has been proposed to study the vibrations of the nanowire. The nonlinear elastic relation concluded from this model is more suitable for the nanowires. With the results of MD simulations and the spring-mass model, the vibration frequency of nanowires is found to increase slightly and nonlinearly with the rise of initial actuation amplitude. On the other hand, based on the MD simulations performed on a number of Fe nanowires, it is found that the ratio between the length and the height of nanowires has a significant influence on vibration behaviors. We define the slender beam and thick beam by the ratio $L / h$. The results from MD simulations signify that the 
slender beam's vibration frequencies are similar to those of Euler-Bernoulli beam. In contrast, the thick beam resembles the Timoshenko beam.

\section{Models and Methods}

\section{Atomistic Simulation.}

In this work, double clamped <100> Fe nanowires are investigated for their vibration properties. We use the Large-scale Atomic/Molecular Massively Parallel Simulator (LAMMPS) [33] to perform these simulations. Fig. 1 shows the model of nanowires used in MD simulations, which is created with iron atoms in positions corresponding to a perfect BCC crystal lattice. The shape of cross-section is chosen to be rectangle. The Fe lattice constant $a$ is chosen as $0.287 \mathrm{~nm}$. For the Fe nanowires, the length $L$ ranges from $20 a$ to $100 a$, while the width $b$ and height $h$ range from $4 a$ to $30 a$. Atoms at both ends of the nanowires are fixed in their positions, which are denoted by "A" in Fig. 1. The rest of the nanowires are free to move. We assume that the vibrations of these Fe nanowires occur in a vacuum. Thus no damping is applied in these simulations. In order to simulate the real nanowire in this scale, the periodic boundary conditions are not imposed in any directions.
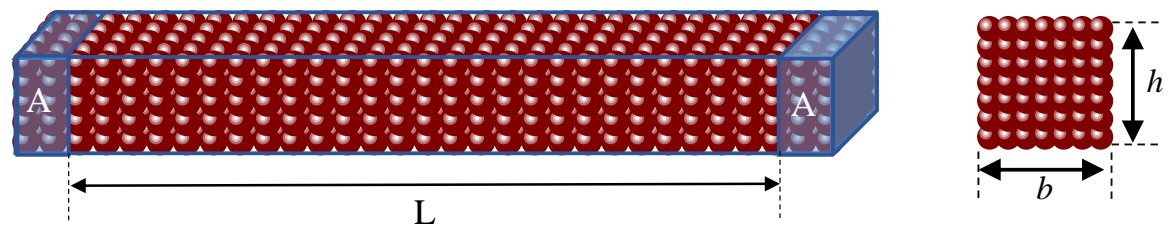

Figure 1. Schematic visualization of a fixed-fixed Fe nanowire used in simulation. The atoms in the areas ' $A$ ' are fixed in all directions and the residual atoms can move freely.

The embedded-atom-method (EAM) potential developed by Mendelev et al [34] is utilized to describe the atomic interactions between $\mathrm{Fe}$ atoms in these simulations, which is a semi-empirical function fitted to a group of parameters, including elastic constants, equilibrium lattice constant, cohesive energy, unrelaxed vacancy formation energy and others. In this model of atomic interaction, the total energy $E_{t o t}$ of a system of $\mathrm{N}$ atoms is a sum of two terms, which are classical pair potential and many-body embedding energy [35].

$$
E_{\text {tot }}=\sum_{i=1}^{N} F_{i}\left(\rho_{i}\right)+\sum_{i=1}^{N} \sum_{j=1}^{N} \phi_{i j}\left(R_{i j}\right)
$$

Here, $F, \rho, \phi$ are the embedded energy, pair potential and electron cloud density. $R_{i j}$ is the 
distance between atom $i$ and $j$.

At the beginning of each simulation, the nanowires are relaxed to the initial equilibrium configuration using the conjugate gradient energy minimization [36]. Then, the Nose-Hoover thermostat $[36,37]$ is employed to equilibrate the nanowires at $0.2 \mathrm{~K}$. Finally, an initial velocity excitation $\mathbf{v}(z)$ is imposed on the nanowires along the $z$-axis.

$$
\mathbf{v}(z)=\lambda \sin \left(\frac{\pi z}{L}\right)
$$

Here, $\lambda$ is actuation amplitude and $L$ is the effective length of the nanowires which exclude the two fixed edges.

\section{Theoretical Models.}

As mentioned before, nanowires are usually utilized as resonating beams in NEMS. According to the classical Euler-Bernoulli beam theory, the governing partial differential equation for the beam is

$$
E I \frac{\partial^{4} w}{\partial x^{4}}+\rho A \frac{\partial^{2} w}{\partial t^{2}}=0
$$

where $E$ is Young's modulus, $I$ is moment of inertia, $\rho$ is density, $A$ is the cross-sectional area of the nanowire, $x$ is position and $t$ is time. $w(x, t)$ is the nanowire's transverse displacement.

For the classical Timoshenko beam theory, the governing partial differential equation is

$$
\left\{\begin{array}{l}
\frac{\partial}{\partial x}\left(E I \frac{\partial \theta}{\partial x}\right)+K G A\left(\theta-\frac{\partial w}{\partial x}\right)-J \frac{\partial^{2} \theta}{\partial t^{2}}=0 \\
\frac{\partial}{\partial x}\left[K G A\left(\theta-\frac{\partial w}{\partial x}\right)\right]+\rho A \frac{\partial^{2} w}{\partial t^{2}}=0
\end{array}\right.
$$

where $G$ is shear modulus, $J$ is moment of inertia per unit length and $K$ is the shear cross-sectional shape factor of the nanowire. $\theta(x, t)$ is the nanowire's rotational angle. After eliminating $\theta$ in Eq. 4, we have

$$
E I \frac{\partial^{4} w}{\partial x^{4}}+\rho A \frac{\partial^{2} w}{\partial t^{2}}-\left(J+\frac{E I \rho}{K G}\right) \frac{\partial^{4} w}{\partial x^{2} \partial t^{2}}+\frac{J \rho}{K G} \frac{\partial^{4} w}{\partial t^{4}}=0
$$

Comparing Eq. 5 with Eq. 3, there are two new items in Eq. 5. These are the effects of shear distortion and rotary inertia, which are considered in Timoshenko model and not considered in Euler-Bernoulli model. 
By applying the clamped-clamped boundary conditions, the vibration frequencies of Euler-Bernoulli beam and Timoshenko beam can be determined by

$$
\begin{gathered}
\omega_{\text {Euler }}=\left(\frac{3}{2} \pi\right)^{2} \sqrt{\frac{E I}{\rho A L^{4}}} \\
\omega_{\text {Timoshenko }}=\left(\frac{3}{2} \pi\right)^{2} \sqrt{\frac{E I}{\rho A L^{4}}} / \sqrt{1+\left(\frac{J}{\rho A}+\frac{E I}{K G A}\right)\left(\frac{3 \pi}{2 L}\right)^{2}}
\end{gathered}
$$

where $L$ is the length of the nanowire.

For the theoretical calculations of the vibration frequency, we define $I=b h^{3} / 12, A=b h$, $K=5(1+v) /(6+5 v) \quad[38,39], \quad J=\rho I$ and $G=E /(2(1+v)) \quad(b$ and $h$ are the width and the height of the nanowire's cross-section, $v$ is the Poisson's ratio) according to the model of the nanowire. For the Fe nanowires, Young's modulus $E$ is chosen as $164 \mathrm{GPa}$ [40], density $\rho$ of $7.87 \mathrm{~g} / \mathrm{cm}^{3}$ and Poisson's ratio $v$ of 0.29 are adopted [41].

To explore the vibrations from the view of lattice structure, we develop a simplified discrete spring-mass model. In classical beam theories, the nanowire is regarded as a continuum. However, with the purpose to show the influence of the atomic arrangement in this scale, the nanowire is seen as a system combined with discrete lattices. We focus our attention on the structure of the cross section. According to the atomic arrangement of the Fe nanowire's cross section, each internal lattice is surrounded by other four lattices as shown in Fig. 2a. Besides, the lattices along the $x$-axis and $y$-axis have the same influence on the internal lattice.

(a)

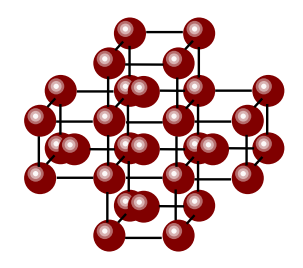

(b)

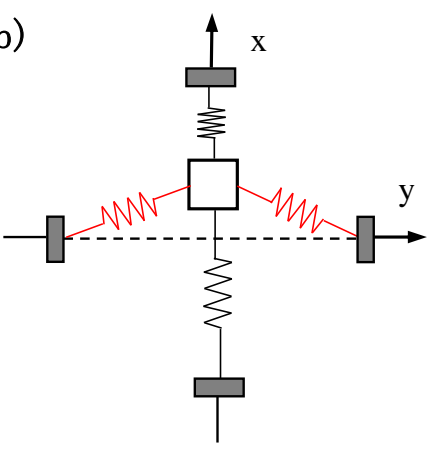

Figure 2. Atomic arrangement and Spring-mass model. (a) The typical atomic arrangement of Fe nanowires with several lattices. (b) Schematic of simplified discrete spring-mass model according to the atomic arrangement of Fe nanowires. It shows the mass move along the $\boldsymbol{x}$-axis. The red springs result in the nonlinearity of 


\section{this model.}

For simplicity, we interpret this structure as a spring-mass system as illustrated in Fig. 2 b. This spring-mass system consists of linear springs, which are defined as

$$
m \ddot{\mathbf{x}}=-k \Delta \mathbf{x}
$$

where $m$ is mass of the object, and $k$ is elastic constant of the spring. Due to the symmetric cross-sectional plane and the same influence along the $x$-axis and $y$-axis, the springs are chosen to be the same. The dynamic equation of this spring-mass system is described as

$$
m \ddot{x}=-4 k x+2 k x \frac{l_{0}}{\sqrt{x^{2}+l_{0}^{2}}}
$$

where $l_{0}$ is the initial length of the spring. In Eq. 9, it is obvious that the second item on the right is a nonlinear item for this equation. As shown in Fig. 2b, when this system vibrates along the $x$-axis, because of the spring-mass model's geometric structure, the red springs produce the nonlinear forces, which correspond to the nonlinear item in Eq. 9. The whole spring-mass system is a kind of nonlinear spring, which may depict the elastic relation of the nanowire more effectively. If $x$ is infinitely small in comparison with $l_{0}$, Eq. 9 will degenerate to the corresponding linear equation as

$$
m \ddot{x}=-2 k x
$$

This is the linear elastic relation we usually use in classical beam theories.

\section{Results and Discussion}

\section{Nanowires in Vibration}

We begin this paper with MD simulations and corresponding analysis on the vibrational Fe nanowires. The external energy is defined as the difference of the potential energy before and after the transverse velocity actuation is applied to the nanowire [21]. We can obtain the energy data directly from the simulations. By analyzing the external energy time history with the Fast Fourier transform (FFT), the vibration frequency and modes of the nanowire can be obtained. In this section, MD simulations are applied to study the Fe nanowire with the size of $20 a \times 10 a \times 100 a$. Fig. 3a depicts the time history of the external energy during the free 
vibration after the initial velocity actuation. Fig. $3 \mathrm{~b}$ presents part of the periodogram regarding the power of the discrete Fourier transformation versus frequency. The whole simulation lasts about 4000 picoseconds with a time step of 1 femtosecond. The amplitude frequency curve highly depends on these data. As seen in Fig. 3b, one main frequency component is identified, which is about $53.41 \mathrm{GHz}$. This result indicates that the Fe nanowire is under a vibration with the frequency of $26.70 \mathrm{GHz}$, because the frequency of the external energy is twice that of the actual vibration.
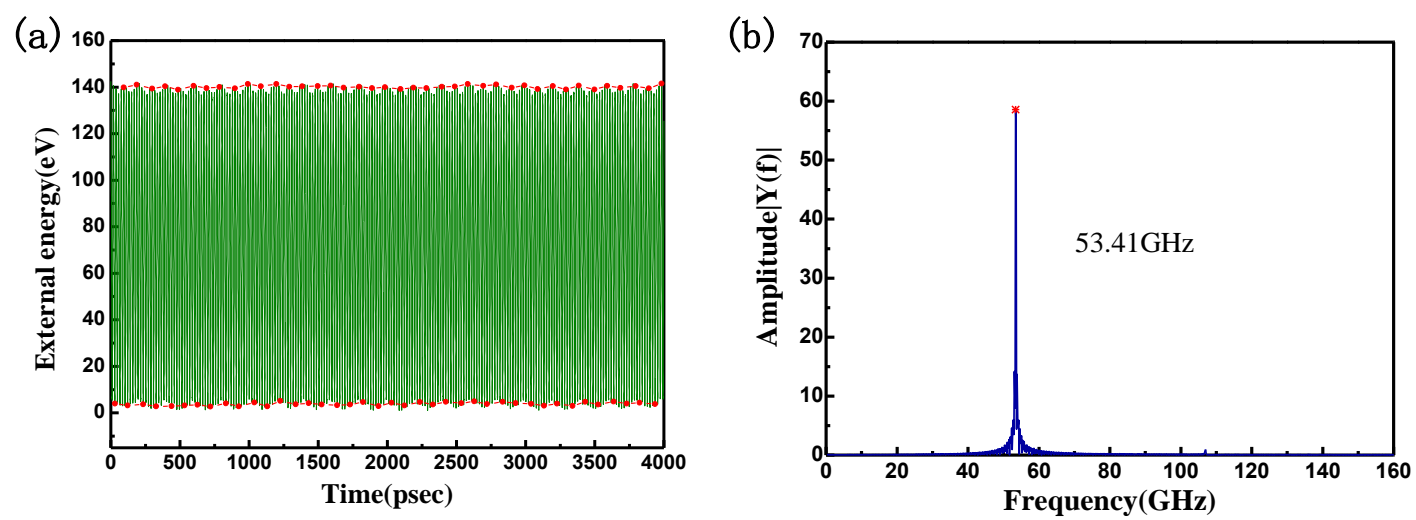

Figure 3. (a) External energy time history of the Fe nanowire with the size of $20 a \times 10 a \times 100 a$ for a free vibration MD simulation at $0.2 K$. The simulation time is from 0 to 4000 picosecond. Circle markers highlight the maximum and minimum of external energy during each vibration circle. (b) The frequency spectrum from FFT analysis on the previous simulation (from 0 to $160 \mathrm{GHz}$ ). The star marker highlights the maximum of the main frequency component.

\section{Initial Actuation Amplitude}

Then, the relation between initial actuation amplitudes and vibration frequencies is studied. We perform MD simulations on the Fe nanowires with the size of $4 a \times 4 a \times 20 a$, $10 a \times 10 a \times 50 a, 5 a \times 5 a \times 50 a$ and $10 a \times 10 a \times 100 a$. Initial velocity excitations as Eq. 2 are imposed on these nanowires. In Eq. 2, several different amplitudes $\lambda$ ranging from 0.2 Angstrom/psec to 1.4 Angstrom/psec are chosen for the tests. Fig. 4 describes the frequencies received from the free vibration $\mathrm{MD}$ simulations of Fe nanowires under different initial actuation amplitudes. As observed in Figs. 4, it is found that the vibration frequency from the MD simulations rises slightly and nonlinearly with the increase of initial actuation amplitude. 

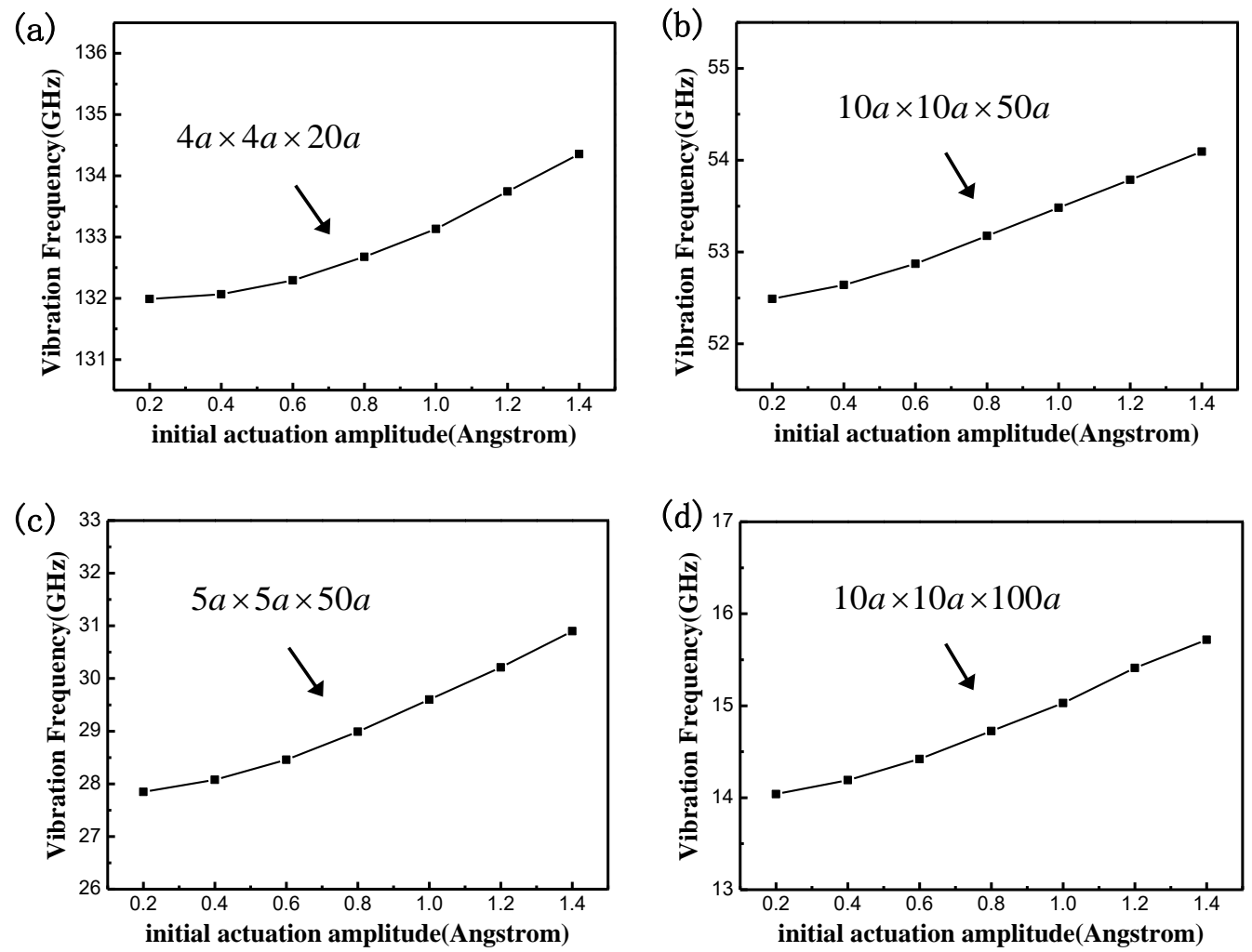

Figure 4. These lines represent the vibration frequencies vs initial actuation amplitude. In this situation, we use the frequencies obtained from FFT analysis when we apply different initial actuation amplitude on different Fe nanowires. They are nanowires with (a) the size of $4 a \times 4 a \times 20 a$. (b) the size of $10 a \times 10 a \times 50 a$. (c) the size of $5 a \times 5 a \times 50 a$. (d) the size of $10 a \times 10 a \times 100 a$.

In addition, we test initial boundary conditions of different actuation amplitudes on the spring-mass model (both the origin nonlinear equation Eq. 9 and the degenerate linear equation Eq. 10). The results are shown in Fig. 5. The nonlinear rise of the black line indicates that with the increase of initial actuation amplitude, the vibration frequency rises slowly, which is similar to the result from the MD simulations. The red line remains constant. It means the vibration frequency is independent to the initial actuation amplitude.

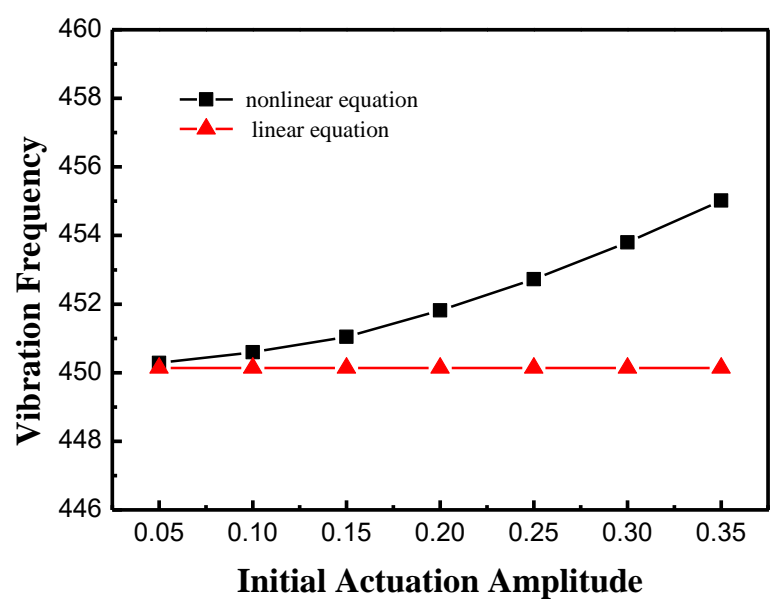


Figure 5. The vibration frequencies vs initial actuation amplitude. In this situation, we apply different initial conditions' value on the spring-mass model. Then, the frequencies can be obtained from FFT analysis on the numerical results. The black line shows the results of Eq. 9 and the red line shows those of Eq. 10.

As shown in Eq. 6 and Eq. 7, both the Euler-Bernoulli beam and Timoshenko beam have fixed natural frequencies, which are irrelevant to initial actuation amplitude. The red line in Fig. 5 has a good agreement with this result. However, both the results from MD simulations and the black line signify that the vibration frequency has a nonlinear correlation with initial actuation amplitude. To explain this phenomenon, we suggest that, in the nano scale, the lattice structure has a big influence on the properties of the nanowires' vibrations. This means the elastic relation of the nanowires may be nonlinear. As mentioned above, according to the geometry structure, the spring-mass model can provide nonlinear elastic relation. The similarity between the results from MD simulations and the black line in Fig.5 shows that the nonlinear elastic relation based on the atomic arrangement may be more suitable for nanowires in this scale.

\section{Length and Height}

Further interest is laid on the relation between nanowires length $(L)$, height $(h)$ and vibration frequencies. In this section, we have done two groups of tests. The nanowires in the first group have the fixed $h$ and the changing $L$, while those in the other group have the fixed $L$ and the changing $h$. Both MD simulations and theoretical calculations have been performed on the two groups. During the MD simulations, the initial actuation amplitude $\lambda$ is fixed at 1.5 Angstrom/psec.

Fig. 6 illustrates the results of the first group. Figs. $6 a$ and $6 \mathrm{~b}$ show the vibration frequency height curve obtained from the MD simulations and Figs. $6 \mathrm{c}$ and $6 \mathrm{~d}$ present the corresponding numerical results of Euler-Bernoulli beam and Timoshenko beam. As observed in Figs. 6, it is obvious that the vibration frequency rises with the increase of height. According to the Euler-Bernoulli beam theory (black lines in Figs. 6c and 6d), the vibration frequency has a linear correlation with the height of the nanowire. However, the vibration frequency in Timoshenko beams (red lines in Figs. 6c and 6d) rises nonlinearly with the increase of $h$. The frequencies of Timoshenko beam are smaller than those of Euler-Bernoulli beam. And their differences become larger with the increase of the height. Comparing the MD results with 
theoretical calculations, we can find that when the value of height is relatively small, the correlation between vibration frequency and height is similar to that of Euler-Bernoulli beam. However, the correlation resembles that of Timoshenko beam when the value of height becomes relatively large. Since $L$ is a fixed value, the ratio between length and height $(L / h)$ declines with the increase of height. We define the slender beams as the nanowires with relatively large value of $L / h$ (usually larger than 5), while the thick beam is defined as the nanowires with relatively large value of $L / h$ (usually smaller than 5). Thus, the observations signify that, in the nano size, the variation of vibration frequencies is similar to Euler-Bernoulli model for the slender beam, while the variation is similar to Timoshenko model for the thick beam.
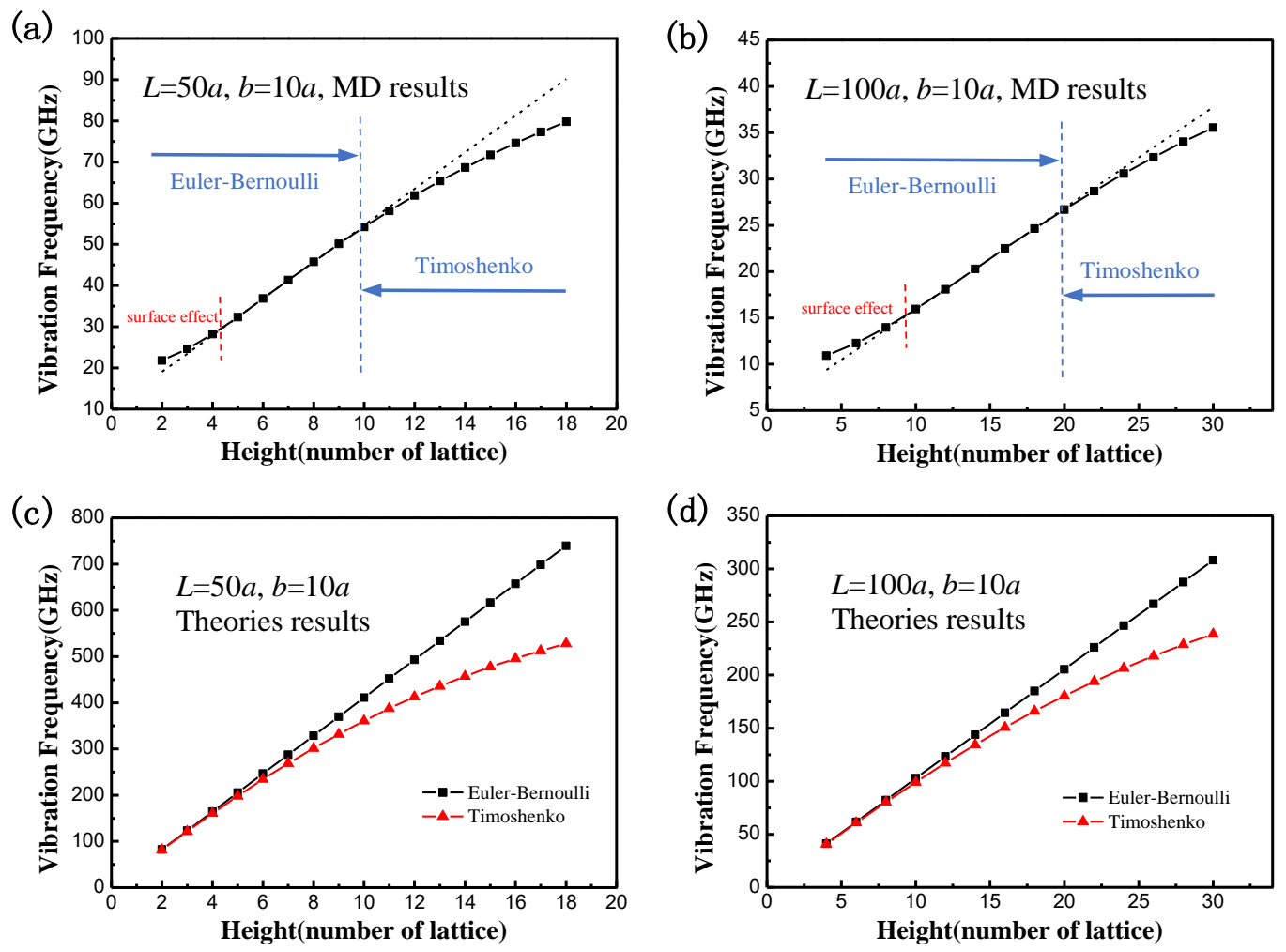

Figure 6. MD results and theoretical results of the first group. (a) and (b) MD results. (c) and (d) Theoretical results. (a) and (c) Nanowires with the fixed $L=50 a, b=10 a$ are tested. (b) and (d) Nanowires with the fixed $L=100 a, b=10 a$ are tested.

It is worth noting that there are small deviations in the beginning of the MD simulations' curves (Figs. 6a and 6b). According to the research by Wang and Feng [38], the surface effects play a significant role in the vibration behavior of nanowire when the height of the nanowires reduces to nanometres. These small deviations accord with their conclusions. Therefore, we suggest that, the small deviations in Figs. $6 \mathrm{a}$ and $6 \mathrm{~b}$ are caused by the surface effects. 
Next, the second group is tested. Figs. 7a and 7c depict the relationship between the length and the vibration frequency, which are received from MD simulations and theoretical calculations on Fe nanowires in different length. From Figs. 7a and 7c, it is found that, in the fixed $h$ and $b$, the vibration frequency declines with the increase of the nanowires' length. In Eq. 6 , there is a linear correlation between vibration frequency and $1 / L^{2}$. Thus, Figs. $7 \mathrm{~b}$ and $7 \mathrm{~d}$ describe the vibration frequency with $1 / L^{2}$. Comparing curves in Figs. $7 \mathrm{~b}$ and $7 \mathrm{~d}$, it is found that the left half curve of MD results is similar to that of Euler-Bernoulli beam, while the right half curve shows the nonlinear trend similar to Timoshenko beam. Since $h$ is fixed at 50a, it is suggested that the correlation between vibration frequency and $1 / L^{2}$ is similar to Euler-Bernoulli model for the slender beam, while the correlation is similar to Timoshenko model for the thick beam.
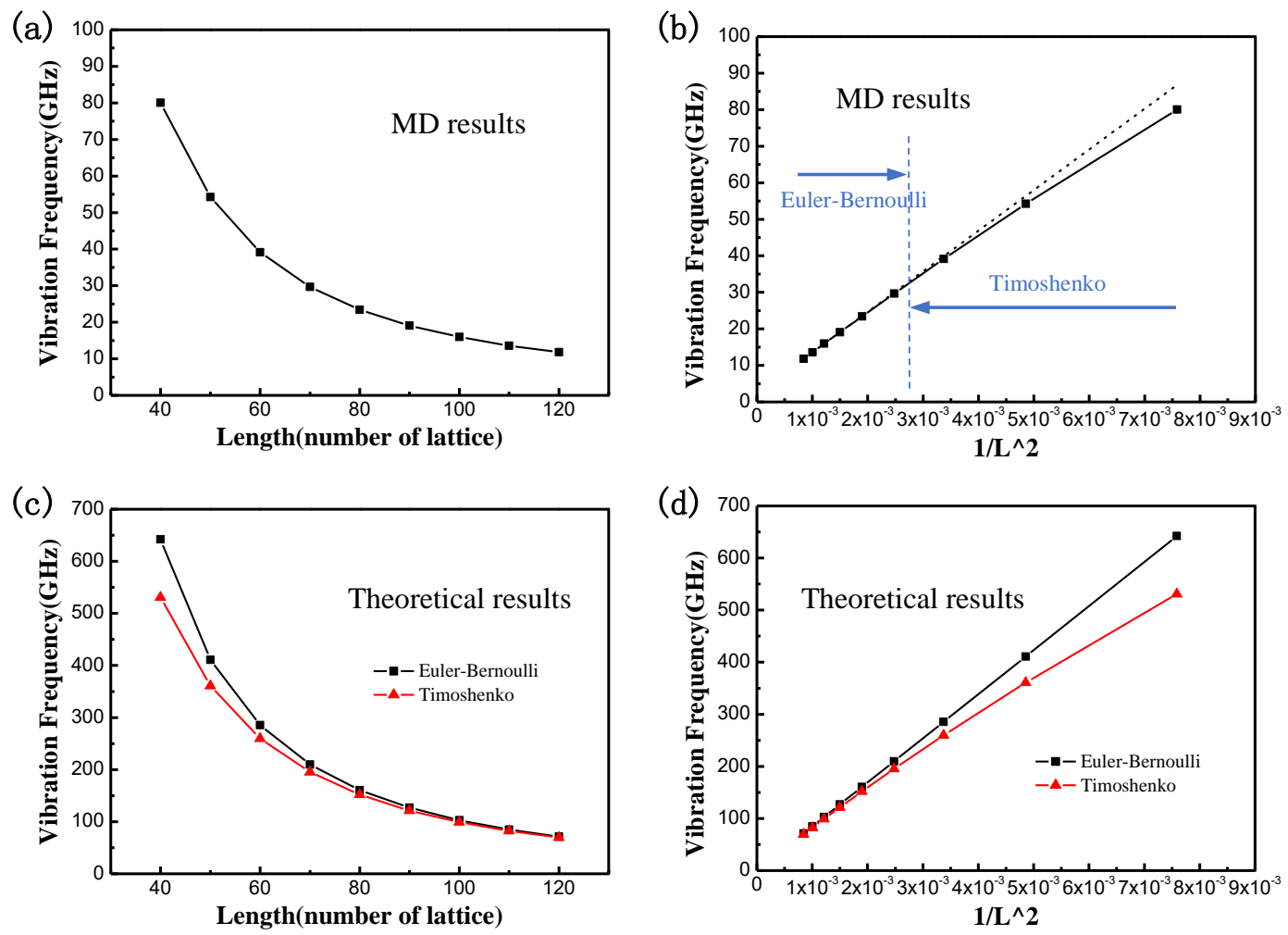

Figure 7. MD results and theoretical results of the second group. In this group, nanowires with the fixed $h=10 a$ and $b=10 a$ are tested. (a) and (b) MD results. (c) and (d) Theoretical results. (a) and (c) The vibration frequencies vs nanowires' length. (b) and (d) The vibration frequencies vs $1 / L^{2}$.

As outlined in Figs. 6 and 7, it is observed that the theoretical results do not accord with the MD results very well. The values of the theoretical calculations are significantly larger than those of MD simulations. We suggest that, the difference between theoretical calculations and 
MD simulations is caused by the size effects. As we know, nanowires in this scale may be influenced by size effects, which are not considered in these theoretical models. However, as analyzed above, the trend in vibration frequency in slender Fe nanowire is similar to that of the Euler-Bernoulli beam, while it is close to the Timoshenko beam in thick beam. Therefore, the MD results of Fe nanowires' vibrations can be explained by these classical theories to some extent.

\section{Conclusion}

In summary, based on large scale molecular dynamics simulations, we investigated nonlinear behaviors in the vibration of $\mathrm{Fe}$ nanowires. It is found that with the increase of initial actuation amplitude, the vibration frequency of nanowires grows slowly and nonlinearly. A discrete spring-mass model abstracted from the atomic arrangement is developed in this work. The nonlinear elastic relation of this model is applied to explore this nonlinear correlation between initial actuation amplitude and vibration frequency, which cannot be explained by the classical beam theories. Furthermore, MD simulations and theoretical calculations on $\mathrm{Fe}$ nanowires with different lengths and heights are performed to show the vibration properties. Fe nanowires with the change of $L$ and $h$ are considered respectively. Both the results show that the variation of vibration frequencies is similar to the Euler-Bernoulli beam for the slender beam, while the variation can be fitted by the Timoshenko beam for the thick beam.

\section{Acknowledgements}

This work was supported in part by The National Natural Science Foundation of China (NSFC) under grant number 11072086, 11372117, J1310022, by the 985 program, by the 211 project of Jilin University, and by Basic funds for science and research in Jilin University. This work was also supported by the Jilin Province Computing Centre through the use of its high-performance computing facilities.

\section{References}

[1] D. Rugar, R. Budakian, H.J. Mamin, B.W. Chui, Single spin detection by magnetic resonance force microscopy, Nature, 430 (2004) 329-332.

[2] T. Stowe, K. Yasumura, T. Kenny, D. Botkin, K. Wago, D. Rugar, Attonewton force detection using ultrathin silicon cantilevers, Applied Physics Letters, 71 (1997) 288-290.

[3] P. Xie, Q. Xiong, Y. Fang, Q. Qing, C.M. Lieber, Local electrical potential detection of DNA by nanowire-nanopore sensors, Nature nanotechnology, 7 (2012) 119-125.

[4] R.-M. Ma, L. Dai, H.-B. Huo, W.-J. Xu, G. Qin, High-performance logic circuits constructed on single CdS 
nanowires, Nano letters, 7 (2007) 3300-3304.

[5] C.K. Chan, H.L. Peng, G. Liu, K. Mcllwrath, X.F. Zhang, R.A. Huggins, Y. Cui, High-performance lithium battery anodes using silicon nanowires, Nature Nanotechnology, 3 (2008) 31-35.

[6] E. Gil-Santos, D. Ramos, J. Martínez, M. Fernández-Regúlez, R. García, Á. San Paulo, M. Calleja, J. Tamayo, Nanomechanical mass sensing and stiffness spectrometry based on two-dimensional vibrations of resonant nanowires, Nature nanotechnology, 5 (2010) 641-645.

[7] M. Li, T.S. Mayer, J.A. Sioss, C.D. Keating, R.B. Bhiladvala, Template-grown metal nanowires as resonators: Performance and characterization of dissipative and elastic properties, Nano Letters, 7 (2007) 3281-3284.

[8] X.-Q. Zeng, Y.-L. Wang, H. Deng, M.L. Latimer, Z.-L. Xiao, J. Pearson, T. Xu, H.-H. Wang, U. Welp, G.W. Crabtree, Networks of ultrasmall Pd/Cr nanowires as high performance hydrogen sensors, Acs Nano, 5 (2011) 7443-7452.

[9] S.Y. Kim, H.S. Park, Utilizing mechanical strain to mitigate the intrinsic loss mechanisms in oscillating metal nanowires, Physical review letters, 101 (2008) 215502.

[10] X. Feng, R. He, P. Yang, M. Roukes, Very high frequency silicon nanowire electromechanical resonators, Nano Letters, 7 (2007) 1953-1959.

[11] H.S. Park, W. Cai, H.D. Espinosa, H. Huang, Mechanics of crystalline nanowires, MRS bulletin, 34 (2009) 178-183.

[12] S.S. Verbridge, J.M. Parpia, R.B. Reichenbach, L.M. Bellan, H. Craighead, High quality factor resonance at room temperature with nanostrings under high tensile stress, J Appl Phys, 99 (2006) 124304.

[13] Y. Yang, C. Callegari, X. Feng, M. Roukes, Surface adsorbate fluctuations and noise in nanoelectromechanical systems, Nano letters, 11 (2011) 1753-1759.

[14] W.G. Conley, A. Raman, C.M. Krousgrill, S. Mohammadi, Nonlinear and nonplanar dynamics of suspended nanotube and nanowire resonators, Nano letters, 8 (2008) 1590-1595.

[15] J. He, C.M. Lilley, Surface stress effect on bending resonance of nanowires with different boundary conditions, Applied physics letters, 93 (2008) 263108.

[16] H.S. Park, Surface stress effects on the resonant properties of silicon nanowires, J Appl Phys, 103 (2008) 123504.

[17] G.F. Wang, X.Q. Feng, Timoshenko beam model for buckling and vibration of nanowires with surface effects, J Phys D Appl Phys, 42 (2009).

[18] Z. Yan, L.Y. Jiang, The vibrational and buckling behaviors of piezoelectric nanobeams with surface effects, Nanotechnology, 22 (2011).

[19] S. Natarajan, S. Chakraborty, M. Thangavel, S. Bordas, T. Rabczuk, Size-dependent free flexural vibration behavior of functionally graded nanoplates, Computational Materials Science, 65 (2012) 74-80.

[20] R. Liu, L. Wang, Vibration of Cantilevered Double-Walled Carbon Nanotubes Predicted by Timoshenko Beam Model and Molecular Dynamics, Int J Comp Meth-Sing, 12 (2015) 1540017.

[21] H.F. Zhan, Y.T. Gu, A fundamental numerical and theoretical study for the vibrational properties of nanowires, J Appl Phys, 111 (2012).

[22] H.F. Zhan, Y.T. Gu, H.S. Park, Beat phenomena in metal nanowires, and their implications for resonance-based elastic property measurements, Nanoscale, 4 (2012) 6779-6785.

[23] J. Zhang, C. Wang, Beat vibration of hybrid boron nitride-carbon nanotubes-A new avenue to atomic-scale mass sensing, Computational Materials Science, 127 (2017) 270-276.

[24] P.A.T. Olsson, Transverse resonant properties of strained gold nanowires, J Appl Phys, 108 (2010).

[25] S.H. Park, J.S. Kim, J.H. Park, J.S. Lee, Y.K. Choi, O.M. Kwon, Molecular dynamics study on size-dependent elastic properties of silicon nanocantilevers, Thin Solid Films, 492 (2005) 285-289.

[26] L.F. Wang, H.Y. Hu, Size Effects on Effective Young's Modulus of Nano Crystal Copper Wires, Int J Comp 
Meth-Sing, 2 (2005) 315-326.

[27] H. Yu, W.W. Zhang, S.Y. Lei, L.B. Lu, C. Sun, Q.A. Huang, Study on Vibration Behavior of Doubly Clamped Silicon Nanowires by Molecular Dynamics, J Nanomater, (2012).

[28] Y.G. Zheng, Y.T. Zhao, H.F. Ye, H.W. Zhang, Size-dependent elastic moduli and vibrational properties of fivefold twinned copper nanowires, Nanotechnology, 25 (2014).

[29] J.-W. Jiang, J.-H. Zhao, T. Rabczuk, Size-sensitive Young's modulus of kinked silicon nanowires, Nanotechnology, 24 (2013) 185702.

[30] J.-W. Jiang, T. Rabczuk, Mechanical oscillation of kinked silicon nanowires: a natural nanoscale spring, Applied Physics Letters, 102 (2013) 123104.

[31] R. Liu, L. Wang, Coupling between flexural modes in free vibration of single-walled carbon nanotubes, AIP Advances, 5 (2015) 127110.

[32] Z. Zheng, E. Li, N. Ding, X. Xu, Beat phenomenon in metal nanowires: A molecular dynamics study, Computational Materials Science, 138 (2017) 117-127.

[33] S. Plimpton, Fast parallel algorithms for short-range molecular dynamics, Journal of computational physics, 117 (1995) 1-19.

[34] M.I. Mendelev, S. Han, D.J. Srolovitz, G.J. Ackland, D.Y. Sun, M. Asta, Development of new interatomic potentials appropriate for crystalline and liquid iron, Philos Mag, 83 (2003) 3977-3994.

[35] M.T. McDowell, A.M. Leach, K. Gall, Bending and tensile deformation of metallic nanowires, Model Simul Mater Sc, 16 (2008).

[36] W.G. Hoover, Canonical dynamics: equilibrium phase-space distributions, Physical review A, 31 (1985) 1695.

[37] S. Nosé, A unified formulation of the constant temperature molecular dynamics methods, The Journal of chemical physics, 81 (1984) 511-519.

[38] G.-F. Wang, X.-Q. Feng, Timoshenko beam model for buckling and vibration of nanowires with surface effects, Journal of physics D: applied physics, 42 (2009) 155411.

[39] H. Zhan, Y. Gu, Modified beam theories for bending properties of nanowires considering surface/intrinsic effects and axial extension effect, J Appl Phys, 111 (2012) 084305.

[40] G. Sainath, B. Choudhary, Orientation dependent deformation behaviour of BCC iron nanowires, Computational Materials Science, 111 (2016) 406-415.

[41] Iron Nanorods, in, American elements, https://www.americanelements.com/iron-nanorods-7439-89-6. 